\title{
Homocysteine level in stroke patients in the rehabilitation setting: a prospective observational study
}

\begin{abstract}
Introduction: Homocysteine (HC), at normal levels, is an important micronutrient in the human body. Elevated $\mathrm{HC}$ may be a risk factor for stroke in the elderly population. Serum levels for HC were used as an index of HC status in thirty patients with stroke. The incidence of hyper-homocysteinaemia was compared with their prognosis and outcome, particularly in terms of the improvement in their function.
\end{abstract}

Objectives: To determine the incidence of hyper-homocysteinaemia in an inpatient population with stroke, in order to identify the linkage of the HC level and the stroke outcome.

Methods: The research was conducted as a prospective data collection and analysis over a period of six months, using a snapshot view of patients with stroke admitted to a tertiary rehabilitation unit. Quantitative measures and analysis were utilized for data analysis. HC level, other inflammatory markers, demographic details and outcome measures were employed to detect the linkage between the hyper-homocysteinaemia and the outcome of stroke.

Results: A total $27 \%$ of the stroke patients in the Rehabilitation Unit demonstrated hyper-homocysteinaemia. Patients with hyper-homocysteinaemia were found having markedly lower cognition as compared to the ones with normal $\mathrm{HC}$ levels.

Conclusion: Incidence of the hyper-homocysteinaemia was $27 \%$ in the patients with stroke in the acute rehabilitation setting. The length of inpatient stay was relatively longer in the patients with hyper-homocysteinaemia as compared to the patients with normal homocysteine levels. These patients also had lower cognition as compared to the patients with normal homocysteine levels.

Keywords: homocysteinaemia, rehabilitation, stroke, serum levels
Volume 2 Issue 3 - 2018

\author{
Ling Lan,' Zeshan Ali \\ 'Geriatric and Rehabilitation Medicine, The Prince Charles \\ Hospital,Australia \\ ${ }^{2}$ Rehabilitation and Pain Services, Cairns Private Hospital, \\ Australia
}

Correspondence: Ling Lan, Geriatric and Rehabilitation Medicine, The Prince Charles Hospital, Rode Road, Chermside, Queensland, QLD 4032, Australia, Tel 07-31395198, Email Ling.Lan@health.qld.gov.au

Received: June 18, 2017| Published: June 27, 2018
Abbreviations: HC, homocysteine; CRP, C-reactive protein; FIM, functional independence measure; MMSE, mini mental status examination; LOS, length of stay

\section{Introduction}

Hyper-homocysteinaemia is an emerging risk factor for ischaemic stroke, ${ }^{1-3}$ but its role in the outcome of the stroke population is controversial. $^{4-8}$ In one study hyper-homocysteinaemia was found in $60.6 \%$ of the patients with ischaemic stroke, which was related to low vitamin $\mathrm{B}_{12}{ }^{4}$ Hyper-homocysteinaemia has been linked to inadequate intake of vitamins and therefore can be amenable to dietary interventions. Poor oral intake of folic acid, vitamin $\mathrm{B}_{6}$ and vitamin $B_{12}$ are linked to an increased risk for ischaemic stroke and cardiovascular disease ${ }^{1}$. Increased intake of fruits and vegetables has a protective role against stroke. ${ }^{9-11}$ Antioxidant nutrients have important roles in the cellular function and have been implicated in the process associated with ageing including vascular, inflammatory and neurological damage. ${ }^{12-17}$ But, there is insufficient evidence to support the routine use of the vitamins for stroke prevention. ${ }^{1}$ Currently, there is little information available on the inflammatory markers such as $\mathrm{HC}$ in the stroke population regarding the outcome. This prospective observational study looks at this issue in order to help us understand the risk factors for stroke and the correlation between the $\mathrm{HC}$ and its linkage with the outcome and prognosis in stroke patients.

\section{Methods}

All identified patients with a diagnosis of stroke, who were admitted to the Rehabilitation Unit over a period of six months in 2010, were included in this study. The inclusion criteria for this study required the patients to be of age forty years and above with a stroke (ischaemic or haemorrhagic). Those with haemorrhagic stroke due to underlying causes other than hypertension were excluded. Data collected included demographic information, risk factors for stroke, usual accommodation, pre-morbid use of walking aids, $\mathrm{HC}$ levels at pre and post treatment, C-reactive protein (CRP) level, Functional Independence Measure (FIM), Mini Mental Status Examination (MMSE), length of stay (LOS), and the discharge destination. The level of $\mathrm{HC}$ was recorded in micromoles/litre (L). The normal value was considered to be $<15$ micromoles/L. The CRP was considered as normal if $\leq 5 \mathrm{mg} / \mathrm{L}$. The fasting blood $\mathrm{HC}$ level was taken in the morning and was analysed by the same laboratory within the hospital campus using the standard protocols and conditions. All identified patients had nutritional assessment by the clinical examination, dietetic assessment and biomarkers such as albumin, vitamin $\mathrm{B}_{12}$ and folate levels. This study was approved by the hospital ethics committee. Data analysis was assessed using a two-sample $t$-test. $\mathrm{P}$ values of less than 0.05 were considered to indicate statistical significance. To address the confounding factor of small sample of group with hyper- 
homocysteinaemia, a matched control analysis was conducted by age (within 4 year) plus MMSE (within 2 points).

\section{Results}

\section{Study population}

The baseline demography of both groups (normal vs. high HC levels) of patients is depicted in the Table 1 . The age of the study population ranged between 42 and 89 years with the mean age of 71.7 years. Out of the total number of 30 patients, 29(97\%) were of the European origin. The remaining 1(3\%) patient was of the Chinese origin. Four $(13 \%)$ out of the thirty patients were from a non-English speaking background. These patients with non-English speaking background had normal $\mathrm{HC}$ levels. Nine (30\%) patients were females and $21(70 \%)$ were males. There was only one patient below 55 years of age, aged 42 years. This patient shared the similar risk factors as the other subjects in this study.

Table I The baseline characteristics.

\begin{tabular}{|c|c|c|}
\hline Characteristics & $\begin{array}{l}\text { High HC } \\
n=8(26.66 \%)\end{array}$ & $\begin{array}{l}\text { Normal HC } \\
n=22(73.33 \%)\end{array}$ \\
\hline Age (years) & $76( \pm 9.18)$ & $70.14( \pm 11.54)$ \\
\hline \multicolumn{3}{|l|}{ Sex } \\
\hline Male & $5(62.5 \%)$ & $16(72.73 \%)$ \\
\hline Female & $3(37.5 \%)$ & $6(27.27 \%)$ \\
\hline BMI & $27.62( \pm 3.81)$ & $27.63( \pm 4.22)$ \\
\hline Language & English & $\begin{array}{c}\text { English: I8(8I.82\%) } \\
\text { Others: } 4(18.2 \%)\end{array}$ \\
\hline \multicolumn{3}{|l|}{ Ethnicity } \\
\hline White & $8(100 \%)$ & $21(95.45 \%)$ \\
\hline Others & $0(0 \%)$ & I(4.54\%) \\
\hline History of $\mathrm{EtOH}$ use & $7(87.5 \%)$ & $20(90.90 \%)$ \\
\hline History of Smoking & $4(50 \%)$ & I7(77.27\%) \\
\hline Family History of stroke & $0(0 \%)$ & $5(22.73 \%)$ \\
\hline Prior IHD & $3(37.5 \%)$ & $8(36.36 \%)$ \\
\hline Prior vascular diseases & $3(37.5 \%)$ & $8(36.36 \%)$ \\
\hline Type 2 Diabetes Mellitus & $3(37.5 \%)$ & $7(31.82 \%)$ \\
\hline Anxiety/depression & $0(0 \%)$ & $7(31.82 \%)$ \\
\hline $\begin{array}{l}\text { Dwelling - } \\
\text { Home }\end{array}$ & $8(100 \%)$ & $21(95.45 \%)$ \\
\hline Marriage & $5(62.5 \%)$ & $12(54.55 \%)$ \\
\hline Walking Aids & $\mathrm{I}(\mathrm{I} 2.5 \%)$ & $4(18.18 \%)$ \\
\hline
\end{tabular}

Data was expressed as mean $\pm S D$. There were no significant differences between the two observational groups at baseline.

$\mathrm{EtOH}$, alcohol; Walking aids, usage of walking aids prior to the stroke; IHD, ischemic heart disease

The HC levels ranged between 7 and 26 micromoles/L. Twenty eight $(93 \%)$ patients had ischaemic stroke and the remaining two (7\%) had an intracranial haemorrhage as a result of hypertension (no underlying vascular aneurysm, arterio-venous malformation, tumour or metastatic brain lesions were identified). All the patients with the haemorrhagic stroke had normal HC levels. These two patients shared the similar risk factors as the patients with the ischaemic stroke. A total of $8(27 \%)$ patients had hyper-homocysteinaemia and the remainder of the $22(73 \%)$ had normal HC levels. Among the eight patients with hyper-homocysteinaemia, six patients received treatment with folic acid. The mean age (76) was higher in the group with high HC levels as compared to the group with the normal $\mathrm{HC}$ levels, mean age of 70.14 . However, there was no statistical significance $(p=0.21)$. Over all, there were a significantly higher number of males as compared to the females in both of these groups. It is of worth noting that in both of high and normal HC groups there was a significant history of smoking. A total of $77 \%$ of the patients in the normal HC group were heavy smokers as compared to $50 \%$ in the group with the high $\mathrm{HC}$ level. There were no significant differences in terms of the BMI, past history of ischaemic heart disease, vascular disease or Diabetes mellitus in both of these groups. However the percentage of the patients with these complications was slightly higher in the hyperhomocysteinaemia group.

Similarly, there were no significant differences in terms of the marital status, premorbid use of walking aids and the community dwelling between both groups. It is interesting to note that a total of seven patients with the diagnosis of anxiety and depression were identified having normal $\mathrm{HC}$ levels. In terms of the functional profile (Table 2A), the mean LOS in the group with high $\mathrm{HC}$ was 63.63 days as compared to 51 days in the group with normal HC (Figure 1). The admission scores of the MMSE for the patients with hyperhomocysteinaemia were significantly lower as compared to the group with the normal HC levels (Figure 2). Similarly, the admission FIM scores were lower in the group with hyper-homocysteinaemia than the other group but showed slightly higher FIM gains as compared to the other group (34.75 vs. 27.09 ) (Figure 3) (Table 2A). The gender distribution was equal in the group with the normal $\mathrm{HC}$ as compared to a slightly higher number of female patients $(37.5 \%$ vs. $27.27 \%)$ in the group with the high $\mathrm{HC}$.

Table 2A Functional profile.

\begin{tabular}{lll} 
Measures & $\mathbf{H i g h} \mathbf{H C}(\mathbf{n}=\mathbf{8})$ & Normal $\mathbf{H C}(\mathbf{n}=\mathbf{2 2})$ \\
\hline MMSE at admission* & $21.50( \pm 4.7)$ & $26.95( \pm 3.2)$ \\
MMSE at discharge* & $21.50( \pm 4.7)$ & $27.04( \pm 3.3)$ \\
GDS(total I5) & $0.50( \pm 0.9)$ & $1.3( \pm 2.7)$ \\
FIM at admission (total 126) & $70.62( \pm 21.0)$ & $81.59( \pm 32.0)$ \\
FIM gain & $34.75( \pm 13.9)$ & $27.09( \pm 18.2)$ \\
FIM efficiency & $0.74( \pm 0.4)$ & $0.66( \pm 0.4)$ \\
LOS (days) & $63.63( \pm 42.9)$ & $51( \pm 36.0)$ \\
Discharge to home rate(\%) & $7(87.5 \%)$ & $20(98.1 \%)$ \\
Discharge support rate & $7(87.5 \%)$ & $17(77.2 \%)$
\end{tabular}

The data was expressed as mean \pm SD. There was significant difference in MMSE between the two observational groups $(* p<0.05)$. No significant differences were observed in other functional profiles.

In terms of the discharge to the community dwelling, $7(87.5 \%)$ patients, with hyper-homocysteinaemia, were discharged to home and one of the inpatients was transferred to other unit and subsequently passed away due to severe pneumonia and renal failure. All of these patients required community supports. On the other hand, from the group with the normal $\mathrm{HC}$ levels, 20(98.1\%) patients were discharged home, $17(77.27 \%)$ required the community support. Due to limited 
participants in the hyper-homocysteinaemia group, it is reasonable to use an age-matched control analysis to limit the multiple confounding factors. Matched control analysis involved matching individual in the hyper-homocysteinaemia group with one from the other group by age within 4 years and MMSE within 2 points was attempted. Unfortunately, only 5 patients were identified within these criteria.

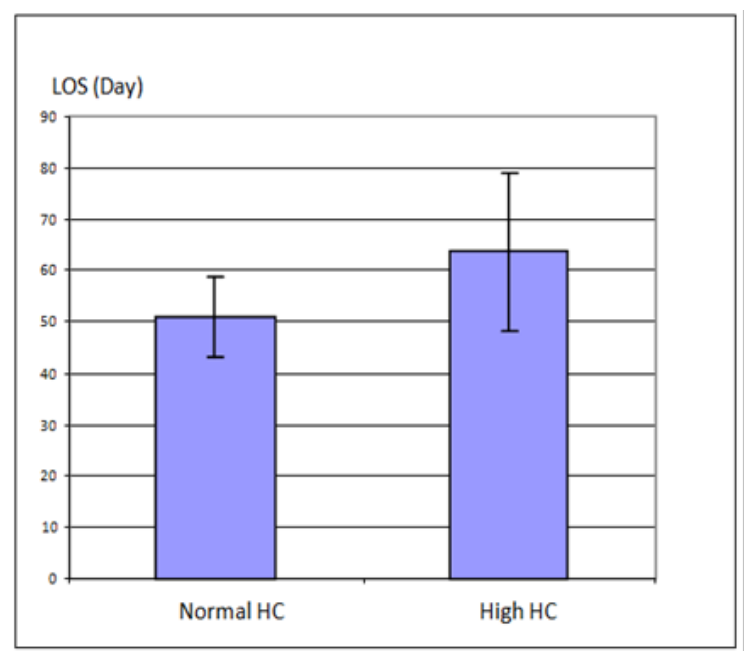

Figure I Length of stay between patients with normal and high HC (the data was expressed as mean \pm SE)

Table 2B Functional profile after age and MMSE matched-control analysis.

\begin{tabular}{lll}
\hline Measures & High HCS(n=5) & $\begin{array}{l}\text { Normal } \\
\text { HCS(n=5) }\end{array}$ \\
\hline Age(years) & $74.6( \pm 10.4)$ & $74.2( \pm 10.5)$ \\
MMSE at admission & $24.4( \pm 2.6)$ & $24.8( \pm 3.4)$ \\
MMSE at discharge & $24.4( \pm 2.6)$ & $24.8( \pm 3.4)$ \\
FIM at admission(total I26) & $68.2( \pm 27.0)$ & $71.6( \pm 36.0)$ \\
FIM gain & $29.4( \pm 15.4)$ & $36.2( \pm 24.1)$ \\
FIM efficiency & $0.52( \pm 0.4)$ & $0.55( \pm 0.3)$ \\
LOS(days) & $78.2( \pm 50)$ & $62.8( \pm 27.7)$ \\
Discharge to home rate(\%) & $4(80 \%)$ & $5(I 00 \%)$ \\
Discharge support rate & 4 out of 4(I00\%) & 4 out of 5(80\%) \\
\hline
\end{tabular}

The data was expressed as mean $\pm S D$. There were no significant differences between the two groups in the functional profiles.

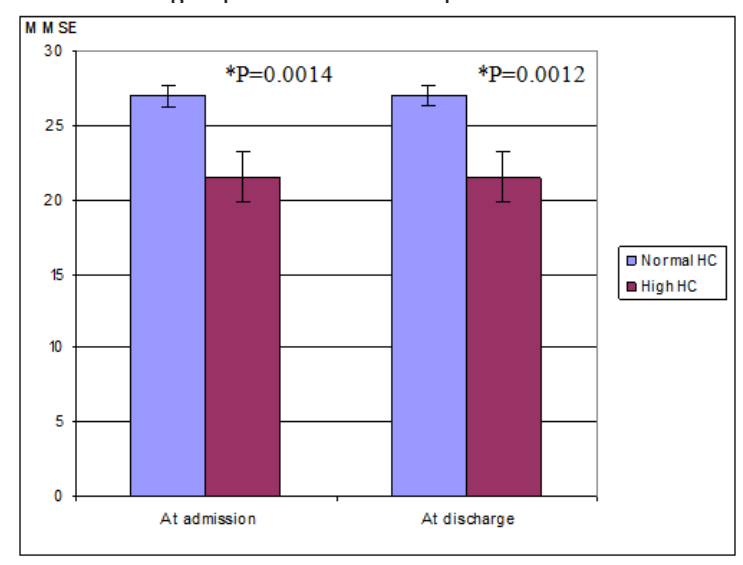

Figure 2 Cognition (MMSE) and level of $\mathrm{HC}$ (the data was expressed as mean $\pm S E)$.

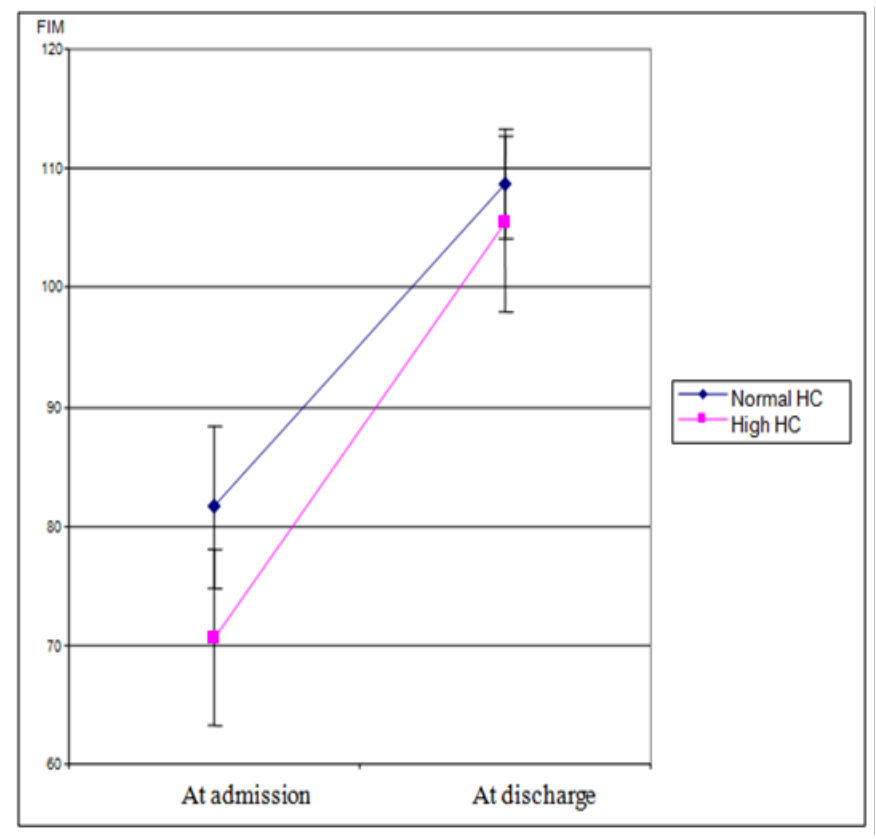

Figure 3 Functional outcome (FIM) at admission and discharge (the data was expressed as mean $\pm \mathrm{SE})$.

As per the figures shown in Table 2B, a moderate difference in functional profile was noticed in terms of the FIM gain, LOS and discharge to home rates, but these differences did not reach statistical significance.

\section{Serum biochemical markers}

The analysis of the nutritional profile suggested that all the parameters assessing nutritional status were within the normal range in both groups, as shown by the serum analysis for vitamin $\mathrm{B}_{12}$, folate and albumin levels (Table 3). There was no significant difference in the levels of CRP in these two groups, which ruled out any other concomitant inflammatory process which could affect the serum levels of these biochemical markers. The levels of $\mathrm{HC}$ improved after treatment with folic acid in the group with hyper-homocysteinaemia. This group also demonstrated improvement of functional status following rehabilitation intervention as shown by FIM score in Table 4.

Table 3 Serum biochemical markers.

\begin{tabular}{lll}
\hline Biochemical markers & $\begin{array}{l}\text { High } \\
\text { HCS(n=8) }\end{array}$ & $\begin{array}{l}\text { Normal } \\
\text { HCS(n=22) }\end{array}$ \\
\hline CRP-mg/L & $9.1( \pm 5.6)$ & $8.5( \pm 7.7)$ \\
Vitamin $B_{12}-\mathrm{pmol} / \mathrm{L}$ & $3 \mid 6.6( \pm|6| . \mathrm{I})$ & $358.6( \pm 277.5)$ \\
Folic acid-nmol/L & $\mid 678.8( \pm 476.2)$ & $125 \mathrm{I} .3( \pm 505.6)$ \\
Albumin-g/L & $36.3( \pm 2.6)$ & $37.6( \pm 2.3)$ \\
\hline
\end{tabular}

The data was expressed as mean $\pm S D$. Normal range of biochemical markers was defined as CRP $\leq 5 \mathrm{mg} / \mathrm{L}$, Vitamin $B_{12}$ I33- $180 \mathrm{pmol} / \mathrm{L}$, Folate $>356 \mathrm{nmol} / \mathrm{L}$, Albumin 35-50g/L. 
Table 4 Functional status pre and post treatment in the high HCS group.

\begin{tabular}{lll}
\hline & Pre treatment & Post treatment \\
\hline HCS & $19.1( \pm 3.2)$ & $15.7( \pm 2.8)$ \\
FIM & $70.6( \pm 21.0)$ & $105.4( \pm 20.7)$
\end{tabular}

The data was expressed as mean $\pm S D$. There were no significant differences in functional status pre and post treatment.

\section{Discussion}

Overall, the incidence of hyper-homocysteinaemia was about $27 \%$ in stroke patients in the acute rehabilitation setting, which was slightly lower as compared to the previous studies ${ }^{4}$. The length of stay for the patients with hyper-homocysteinaemia was longer as compared to the patients with a normal level. Similarly, the admission FIM was lower for the group with the high $\mathrm{HC}$ level than the group with the normal HC. Although, the normal HC group demonstrated higher FIM, shorter length of stay, and higher independence and rate of returning home, there were no statistically significant differences in functional profile. The limited sample size may attribute to the non-significant statistical finding.

This study aims to address the question of the association of hyperhomocysteinaemia with rehabilitation outcomes. However, the results are confounded by the greater cognitive impairment and greater age of the hyper-homocysteinaemia group. Due to the limited sample size in hyper-homocysteinaemia group, we were unable to apply a linear regression analysis. Therefore, an age-matched control analysis was applied between hyper-homocysteinaemia participants and control group with normal $\mathrm{HC}$ level. When the match-analysis by both age and MMSE was performed, there were no significant statistical differences in most of the functional profile. There was non-statistical significance of moderate improvement in LOS in normal HC group compared to the high-HC group. However, due to limited sample size, the results remain inconclusive.

This study provides us the evidence of lower cognition in the patients with a higher homocysteine level as compared to the patients with a normal $\mathrm{HC}$ value. This evidence can be linked to cognitive decline as a result of hyper-homocysteinaemia, as per previous studies. ${ }^{8,18,19}$ The mean age difference in the patients between the two groups was of about 6 years (older age in the patients with hyperhomocysteinaemia); however, there is no statistically significant difference. A matched control analysis showed less difference in cognition in these two groups, which may due to limited number of patients. Therefore, the lower cognition in the patients with the higher $\mathrm{HC}$ levels cannot be fully excluded from the association of the age related decline in our limited study. Further studies are needed to elaborate this association.

The average age was 71.7 years. Only one patient aged 42 years shared the similar risk factors as the other older subjects. Therefore, it is considered very unlikely that this would skew of our data. The normal results for the biochemical profile to assess for malnutrition ruled out malnutrition and poor general health as a confounding factor in this study. There are a few limitations in this study. The sample size of the present study is small. The effects of treatment on the long term outcomes for the patients who received treatment for hyperhomocysteinaemia are not available. There is no long term follow up organised for the subjects in this study. Given very limited number of patients with hemorrhagic stroke, we did not separate our data between patients with ischaemic stroke from hemorrhagic stroke. This may produce some potential flaw of our data. Broader scale studies are needed to exclude and evaluate the pre-morbid environmental and personal factors that likely contribution to hyper-homocysteinaemia e.g. poor nutritional status, chronic disease, and alcohol abuse. Furthermore, a larger analysis of randomized controlled study is worthwhile to be considered to better define the potential adverse association of the hyper-homocysteinaemia and function.

\section{Conclusion}

Hyper-homocysteinaemia is associated with ischaemic cerebrovascular and cardiovascular disease. Over all, the incidence of hyper-homocysteinaemia in the patients with stroke was about $27 \%$ in this acute rehabilitation setting which was slightly lower than the previous studies. The length of stay for the patients with hyperhomocysteinaemia was relatively longer as compared to the patients with a normal level of HC. Although, the group with the normal HC levels demonstrated higher FIM, shorter length of stay, higher independence and rate of returning home, there were no statistically significant differences between the two groups. In this study, we found some level of evidence of lower cognition in the patients with a higher $\mathrm{HC}$ level as compared to the patients with a normal $\mathrm{HC}$ value. In other words, our data demonstrate that higher homocysteine levels are associated with impaired cognition, as previously reported by others. ${ }^{8,19,20}$ Further large-scaled studies are needed to elaborate the association between the lower cognition and functional outcomes of hyper-homocysteinaemia with and without treatment.

\section{Acknowledgments}

We thank Mrs. Kay Lehman for her assistance with data collection.

\section{Conflict of interests}

The author declares no conflict of interest.

\section{References}

1. Perry IJ. Homocysteine and Risk of Stroke. J Cardiovasc Risk. 1999;6(4):235-240.

2. Hankey GJ, Eikelboom JW. Homocysteine and Stroke. Curr Opin Neurol. 2001;14(1):95-102.

3. Pezzini A, Del Zotto E, Padovani A. Homocysteine and cerebral ischaemia: Pathogenic and Therapeutic implications. Curr Med Chem. 2007;4(3):249-263

4. Sanchez-Moreno C, Jimenez-Escrig A, Martin A. Roles of B vitamins, homocysteine and antioxidants. Nutr Res Rev. 2009;22(1):49-67.

5. Kalita J, Kumar G, Bansal V, et al. Relationship of homocysteine with other risk factors and outcome of ischemic stroke. Clin Neurol Neurosurg. 2009;111(4):364-367.

6. Terwecoren A, Steen E, Benoit D, et al. Ischemic stroke and hyperhomocysteinemia: truth or myth? Acta Neurol Belg. 2009;109(3):181-188.

7. Hankey GJ. Is plasma homocysteine a modifiable risk factor for stroke? Nat Clin Pract Neurol. 2006;2(1):26-33.

8. Homocystein studies collaboration. Homocysteine and risk of ischemic heart disease and stroke: a meta-analysis. JAMA. 2002;288(16):20152022 . 
9. Acheson RM, Williams DR. Does consumption of fruit and vegetables protect against stroke. Lancet. 1983; 1(8335):1191-1193.

10. Sarkar PK, Lambert LA. Aetiology and treatment of hyperhomocysteinaemia causing ischaemic stroke. Int J Clin Pract. 2001;55(4):262-268.

11. Ascherio A, Rimm EB, Hernan MA, et al. Relation of consumption of vitamin $\mathrm{E}$, vitamin $\mathrm{C}$, and carotenoids to risk for stroke among men in the United States. Ann Int Med. 1999;130(12):963-970.

12. Gonzalez-Gross M, Marcos A, Pietrzik K. Nutrition and cognitive impairment in the elderly. Br J Nutr. 2001;86(3):313-332.

13. Singleton CK, Martin PR. Molecular mechanisms of thiamine utilization. Curr Mol Med. 2001;1(2):197-207.

14. Nilsson K, Gustafson L, Hultberg B. Plasma homocysteine is a sensitive marker for tissue deficiency of both cobalamines and folates in a psychogeriatric population. Dement Geriatr Cogn Disord. 1999; 10(6):476-482.
15. Miller JW, Green R, Mungas DM, et al. Homocysteine, vitamin $\mathrm{B}_{6}$, and vascular disease in AD patients. Neurology. 2002;58(10):1471-1475.

16. Rogers PJ. A healthy body, a healthy mind: long-term impact of diet on mood and cognitive function. Proc Nutr Soc. 2001;60(1):135-143.

17. Toole JF, Malinow MR, Chambless LE, et al. Lowering homocysteine in patients with ischemic stroke to prevent recurrent stroke, myocardial infarction, and death: the Vitamin Intervention for Stroke Prevention (VISP) randomized controlled trial. JAMA. 2004;291(5):565-575.

18. Patel MD, Coshall C, Rudd AG, et al. Cognitive impairment after stroke: clinical determinants and its associations with long-term stroke outcomes. $J$ Am Geriatr Soc. 2002;50(4):700-706.

19. Lehmann M, Gottfries CG, Regland B. Identification of cognitive impairment in the elderly: homocysteine is an early marker. Dement Geriatr Cogn Disord. 1999;10(1):12-20.

20. Wang HX, Wahlin A, Basun $\mathrm{H}$, et al. Vitamin $\mathrm{B}_{12}$ and folate in relation to the development of Alzheimer's disease. Neurology. 2001;56(9):11881194 . 